\title{
NRQCD on an anisotropic lattice *
}

\author{
I.T. Drummond R.R. Horgan T. Manke H.P. Shanahan ${ }^{\text {a }}$

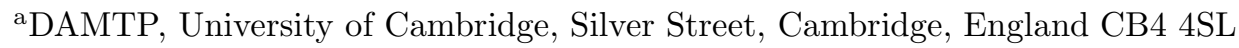

We present preliminary results for the $\Upsilon$ spectrum on an anisotropic lattice using the improved $O\left(m v^{6}\right)$ NRQCD Hamiltonian. We find that accurate results can be obtained in moderate computer times and that they agree with earlier results on an isotropic lattice.

\section{The anisotropic lattice}

On an anisotropic lattice an improved action is used in which the coupling in the "time" direction is tuned so that the temporal lattice spacing $a_{t}$ is much smaller than the spatial lattice spacing $a_{s}$, thus allowing refined measurements of mass differences whilst allowing a coarse spatial lattice. The bare anisotropy $\chi$ is a parameter in the action which determines the renormalized anisotropy $\chi_{R}=a_{s} / a_{t}$. We use the improved action given by [1, 2]:

$$
\begin{aligned}
& S_{n}=-\beta \sum_{x, s>s^{\prime}} \chi^{-1}\left\{\frac{5}{3} \frac{P_{s, s^{\prime}}}{u_{s}^{4}}-\frac{1}{12} \frac{R_{s s, s^{\prime}}}{u_{s}^{6}}-\right. \\
& \left.\frac{1}{12} \frac{R_{s^{\prime} s^{\prime}, s}}{u_{s}^{6}}\right\}-\beta \sum_{x, s} \chi\left\{\frac{4}{3} \frac{P_{s, t}}{u_{s}^{2} u_{t}^{2}}-\frac{1}{12} \frac{R_{s s, t}}{u_{s}^{4} u_{t}^{2}}\right\} .
\end{aligned}
$$

Here $s, s^{\prime}$ run over spatial directions, $P_{s, s^{\prime}}$ is a $1 \times 1$ plaquette, $R_{s^{\prime} s^{\prime}, s}$ is a $2 \times 1$ plaquette, and $u_{s}$ and $u_{t}$ are tadpole improvement parameters which are determined self-consistently. The value of $\chi_{R}$ is measured, for example, by computing the static quark potential in both the coarse and fine directions and we suggest below an alternative method to measure $\chi_{R}$. We use lattices whose parameters were given to us by Alford et al.: $\beta=$ $1.8, \chi=4, \chi_{R}=3.815(10), u_{s}=0.7255, u_{t}=$ 0.9812 , where the tadpole coefficients are given by the respective mean link values in the Landau gauge. Typical lattice sizes used were $8^{3} \times 40$ and $6^{3} \times 60$.

*Presented by R.R. Horgan. This work is supported in part by the Leverhulme Trust grant no. F618C and by PPARC Research Grant GR/L56039.
Whilst we have worked with the values given above for $u_{s}$ and $u_{t}$ we have also implemented a fourier accelerated self-consistent calculation of the Landau gauge definition of these parameters. The definition used for Landau gauge is to maximize

$\sum_{x \mu} \frac{1}{u_{\mu} a_{\mu}^{2}} \operatorname{Re} \operatorname{Tr}\left\{U_{\mu}(x)-\frac{1}{16 u_{\mu}} U_{\mu}(x) U_{\mu}(x+\hat{\mu})\right\}$.

with respect to gauge transformations, and define $u_{\mu}=\left\langle U_{\mu}\right\rangle_{\text {Landau. }}$. this procedure is very timeconsuming. For example, on $8^{3} \times 32$ to fix the gauge to 1 in $2 \cdot 10^{-4}$ requires between 50 and 200 iterations taking, on average, 30 minutes on a $533 \mathrm{MHz}$ DEC PC. To obtain better than $1 \%$ accuracy requires about 50 such measurements. We have made preliminary determinations of $u_{s}$ and $u_{t}$ on $8^{3} \times 32$ and $4^{3} \times 16$ and find a lattice size dependence:

$4^{3} \times 16: \quad u_{s}=0.680(2), u_{t}=0.9792(3)$,

$8^{3} \times 32: u_{s}=0.707(3), u_{t}=0.9811(2)$.

The $8^{3}$ values are close to those of Alford et al. and the discrepancy should have little effect on our results.

\section{NRQCD to $O\left(m v^{6}\right)$}

In NRQCD the inversion problem of the fermion matrix is an initial value problem. We use the evolution equation along the Euclidean time-direction defined by:

$$
\begin{aligned}
& G(\boldsymbol{x}, t+1 ; \boldsymbol{y})= \\
& \quad \mathcal{U}(n, t)\left(1-a_{t} \delta H\right) G(\boldsymbol{x}, t ; \boldsymbol{y}), \quad t>0 \\
& G(\boldsymbol{x}, 1 ; \boldsymbol{y})=\mathcal{U}(n, 0) S(\boldsymbol{x}, \boldsymbol{y}),
\end{aligned}
$$


$\mathcal{U}(t, n)=\left(1-\frac{a_{t} H_{0}}{2 n}\right)^{n} U_{t}^{\dagger}\left(1-\frac{a_{t} H_{0}}{2 n}\right)^{n}$.

Here $S(\boldsymbol{x}, \boldsymbol{y})$ is the source at the first timeslice, $t=0$. We have $S(\boldsymbol{x}, \boldsymbol{y})=\delta^{(3)}(\boldsymbol{x}, \boldsymbol{y})$ for a single quark at the origin but we also evolve a quark with a smeared source centred at $\boldsymbol{y}$. For smearing on a length scale $l$ the extended source is defined by

$S(\boldsymbol{x}, \boldsymbol{y} ; l)=\left(1+\frac{l^{2} \mathbf{D}^{2}}{4 m}\right)^{m} \delta^{(3)}(\boldsymbol{x}, \boldsymbol{y})$,

where $\mathbf{D}$ is the covariant derivative on $\boldsymbol{x}$. Typically, $l=1, m=10$. To construct $P$-wave meson states we also need single quark propagators evolved from a source of the form

$P_{i}(\boldsymbol{x}, \boldsymbol{y} ; l)=\left(1+\frac{l^{2} \mathbf{D}^{2}}{4 m}\right)^{m} D_{i} \delta^{(3)}(\boldsymbol{x}, \boldsymbol{y})$.

The NRQCD Hamiltonian to $O\left(m v^{6}\right)$ is given by $[3$, 目]

$$
\begin{aligned}
H_{0} & =-\frac{\Delta^{2}}{2 m_{b}}, \\
\delta H & =-c_{0} \frac{\Delta^{4}}{8 m_{b}^{3}}-c_{1} \frac{1}{2 m_{b}} \sigma \cdot g \mathbf{B} \\
& +c_{2} \frac{i}{8 m_{b}^{2}}(\Delta \cdot g \mathbf{E}-g \mathbf{E} \cdot \Delta) \\
& -c_{3} \frac{1}{8 m_{b}^{2}} \sigma \cdot(\tilde{\Delta} \times g \mathbf{E}-g \mathbf{E} \times \tilde{\Delta}) \\
& -c_{4} \frac{1}{8 m_{b}^{3}}\left\{\Delta^{2}, \sigma \cdot g \mathbf{B}\right\} \\
& -c_{5} \frac{3}{64 m_{b}^{3}}\left\{\Delta^{2}, \sigma \cdot(\Delta \times g \mathbf{E}-g \mathbf{E} \times \Delta)\right\} \\
- & c_{6} \frac{i}{8 m_{b}^{3}} \sigma \cdot g \mathbf{E} \times g \mathbf{E} \\
- & c_{7} \frac{a_{s} \Delta^{4}}{16 n \chi_{R} m_{b}^{2}}+c_{8} \frac{a_{s}^{2} \Delta^{(4)}}{24 m_{b}} .
\end{aligned}
$$

The fields $\mathbf{E}$ and $\mathbf{B}$ are derived from the improved field strength tensor

$F_{\mu \nu}=F_{\mu \nu}^{(c l)}-\frac{1}{6}\left(a_{\mu}^{2} D_{\mu}^{2}+a_{\nu}^{2} D_{\nu}^{2}\right) F_{\mu \nu}^{(c l)}$,

where $F_{\mu \nu}^{(c l)}$ is the standard clover definition. We set $c_{i}=1, i=0, \ldots, 8$.
In this preliminary study we calculate the lowlying $S$-wave triplet and singlet states $P$-wave singlet states.

For $S$-wave states we use the propagators

$$
\begin{aligned}
& \text { singlet: } \quad \Gamma_{a}^{S}(\boldsymbol{p}, t ; \boldsymbol{y})= \\
& \sum_{\boldsymbol{x}} \cos (\boldsymbol{p} \cdot \boldsymbol{x}) \operatorname{Re} \operatorname{Tr}\left(G_{a}^{S \dagger}(\boldsymbol{x}, t ; \boldsymbol{y}) G_{1}^{S}(\boldsymbol{x}, t ; \boldsymbol{y})\right), \\
& \text { triplet: } \quad \Gamma_{a}^{S}(\boldsymbol{p}, t ; \boldsymbol{y})= \\
& \sum_{\boldsymbol{x}} \cos (\boldsymbol{p} \cdot \boldsymbol{x}) \operatorname{Re} \operatorname{Tr}\left(\boldsymbol{\sigma} G_{a}^{S \dagger}(\boldsymbol{x}, t ; \boldsymbol{y}) \boldsymbol{\sigma} G_{1}^{S}(\boldsymbol{x}, t ; \boldsymbol{y})\right),
\end{aligned}
$$

where $G^{S}$ is evolved with a source defined by eqn. 2, where $\boldsymbol{p}$ is an appropriate lattice momentum and where the subscripts label different initial sources. In practice, we considered only $a=1$ corresponding to the local delta-function source and $a=2$ corresponding to smearing by $l=a_{s}$.

For $P$-wave states we use the propagator

$$
\begin{aligned}
& \Gamma_{a}^{P}(\boldsymbol{p}, t ; \boldsymbol{y})= \\
& \quad \sum_{\boldsymbol{x}} \operatorname{Re} \operatorname{Tr}\left(G_{i, a}^{P \dagger}(\boldsymbol{x}, t ; \boldsymbol{y}) \cdot D_{i} G_{a}^{S}(\boldsymbol{x}, t ; \boldsymbol{y})\right),
\end{aligned}
$$

where $G^{P}$ has been evolved from a source defined by eqn. 3 with label $i$ denoting the spatial derivative. Again, we choose $a=1,2$ defined as for the $S$-wave states. The averaged propagators are fitted by a full two- or three-exponential fit using the standard SVD technique with full correlation matrix to give the various mass differences. The value of $a_{t}$ is determined from the $1^{1} P_{1}-1{ }^{3} S_{1}$ mass difference using the experimental value of $440 \mathrm{MeV}$ and then $a_{s}=a_{t} / \chi_{R}$.

In the case of the $1^{3} S_{1}$ state the kinetic mass, $M_{k}$, is determined by fitting the exponent of the leading exponential term to

$E_{S}(\boldsymbol{p})=E_{S}(0)+p^{2} / 2 M_{k}+\left(p^{2}\right)^{2} / 8 M_{k}^{3}$.

The natural unit in the simulation for $M_{k}$ is $a_{t} / a_{s}^{2}=1 /\left(a_{t} \chi_{R}^{2}\right)$. The bare quark mass entering the definition of $H$, eqn. 1 , is chosen so that this kinetic mass agrees with the experimental $\Upsilon$ mass. In terms of the computed dimensionless kinetic mass, $\bar{M}_{k}$, we have $M_{k}=\bar{M}_{k} / a_{t} \chi_{R}^{2}$. This equation yields a method for measuring the renormalized anisotropy $\chi_{R}$. Given the dimensionless kinetic masses for the these states, $\bar{M}_{k}^{S}$ and $\bar{M}_{k}^{P}$ respectively, we find

$\chi_{R}^{2}=\left(\bar{M}_{k}^{P}-\bar{M}_{k}^{S}\right) /\left(\bar{E}_{P}-\bar{E}_{S}\right)$, 
Table 1

\begin{tabular}{ccccccc}
\hline$L$ & $m_{b} a_{t}$ & $\left(1^{1} P_{1}-1^{3} S_{1}\right) a_{t}$ & $a_{t}^{-1} \mathrm{MeV}$ & $a_{s}^{-1} \mathrm{MeV}$ & $M_{k} \chi^{2} a_{t}$ & $M_{k} \mathrm{MeV}$ \\
\hline 6 & 1.5 & $0.1453(8)$ & $3028(16)$ & $794(5)$ & $50.05(16)$ & $10413(100)$ \\
8 & 1.5 & $0.1467(40)$ & $2999(80)$ & $786(20)$ & $48.4(1)$ & $9973(300)$ \\
8 & 1.7 & $0.1584(40)$ & $2780(70)$ & $723(20)$ & $58.5(2)$ & $11174(250)$ \\
8 & 1.9 & - & - & - & $68.7(2)$ & $13122(250)$ \\
\hline
\end{tabular}

Table 2

\begin{tabular}{llcccc}
\hline & $\mathrm{L}$ & $\Delta E a_{t}$ & $\Delta E \mathrm{MeV}$ & $\Delta E$ (isotropic) $\mathrm{MeV}$ & $\Delta E$ (expt.) $\mathrm{MeV}$ \\
\hline $1^{3} S_{1}-1^{1} S_{0}$ & 6 & $0.00598(2)$ & $18.11(7)$ & $26.1(1)$ & - \\
& 8 & $0.0060(3)$ & $18.0(1.0)$ & & - \\
$2{ }^{1} S_{0}-1^{1} S_{0}$ & 6 & $0.190(4)$ & $575(12)$ & $610(50)$ & \\
& 8 & $0.185(4)$ & $554(20)$ & & 560 \\
$2{ }^{3} S_{1}-1^{3} S_{1}$ & 6 & $0.185(3)$ & $560(10)$ & $560(50)$ & \\
& 8 & $0.186(4)$ & $558(20)$ & & \\
$1^{1} P_{1}-1^{3} S_{1}$ & 6 & $0.1453(8)$ & 440 & 440 & 775 \\
& 8 & $0.1467(40)$ & & & \\
$2{ }^{1} P_{1}-1^{3} S_{1}$ & 6 & $0.37(1)$ & $1120(33)$ & - & \\
\hline
\end{tabular}

where $\bar{E}_{P}$ and $\bar{E}_{S}$ are the dimensionless exponents of the leading decay in each case.

The gauge field configurations were generated by using Cabibbo-Marinari and microcanonical updates. The simulation was carried out on the Hitachi SR2201 parallel computer at the University of Cambridge High Performance Computing Facility. An 8 hour job produced about 2500 independent configurations and propagator.

\section{Results and Conclusion}

In Table 1 we show the results on a $L^{3} \times T$ lattice for the $1^{3} S_{1}$ kinetic mass as a function of $m_{b}$ (eqn. 4 ) and the determination of the lattice spacing from $1^{1} P_{1}-1^{3} S_{1}$. We have not computed the spectrum for $m_{b}=1.9$ but the lattice spacings should change only little from the values quoted for the other masses. We worked with $m_{b}=1.5 a_{t}^{-1}$ for which $M_{k}$ is a bit high but tolerable. Our value for $a_{s}^{-1} \sim 800 \mathrm{MeV}$ agrees well with the value communicated to us by Alford et al. The $S$ and $P$ state mass differences in the various cases are given in Table 2. In the second to last column are the results from our high statistics analysis of UKQCD quenched isotropic lattice configurations on $16^{3} \times 48$ at $\beta=6.0$ [ 5]. A combined fit to the $1^{3} S_{1}$ and $1^{1} S_{0}$ data gives the triplet-singlet mass difference. The fits to both $S$ and $P$ wave data are very good.

Our preliminary study of NRQCD using an improved action on an anisotropic lattice has shown that the results for heavy quark states agree well with similar studies using a standard action on isotropic lattices of smaller spatial lattice spacing, and the accuracy with which parameters can be measured with modest amounts of computer time is superior. There is no lattice size dependence in changing from $L=8$ to $L=6$.

\section{REFERENCES}

1. C. Morningstar. Nucl. Phys. B (proc. Suppl.), 53:914, (1996). Proceeding LATTICE '96.

2. M. Alford et al. Nucl. Phys., B496:377-407, (1997).

3. G.P. Lepage et al. Phys. Rev., D46:4052, (1992).

4. C. Morningstar. Phys. Rev., D48:2265, (1993).

5. I.T. Drummond et al. Phys. Lett., B408:308314, (1997). 